\title{
Well-posedness of the stochastic transport equation with unbounded drift.
}

\author{
David A.C. Mollinedo ${ }^{*}$ Christian Olivera $^{\dagger}$
}

Key words and phrases. Stochastic partial differential equation, transport equation, Low regularity, Stochastic characteristic method.

MSC2010 subject classification: 60H15, 35R60, 35F10, 60H30.

\begin{abstract}
The Cauchy problem for a multidimensional linear transport equation with unbounded drift is investigated. Provided the drift is Holder continuous, existence, uniqueness and strong stability of solutions are obtained. The proofs are based on a careful analysis of the associated stochastic flow of characteristics and techniques of stochastic analysis.
\end{abstract}

\section{Introduction}

We consider the deterministic linear transport equation in $\mathbb{R}^{d}$

$$
\partial_{t} u(t, x)+b(t, x) \cdot \nabla u(t, x)=0,
$$

this equation is one of the most fundamental and at the same time most elementary partial differential equation with applications in a wide range of problems from physics, engineering, biology or social science.

When the coefficients are regular the unique solution is found by the method of characteristics. Recently research activity has been devoted to

\footnotetext{
*Universidade Tecnológica Federal do Parana, Brazil. E-mail: davida@utfpr.edu.br

†Departamento de Matemática, Universidade Estadual de Campinas, Brazil. E-mail: colivera@ime.unicamp.br.
} 
study continuity/transport equations with rough coefficients, showing a wellposedness result. Di Perna andLions [8] have introduced the notion of renormalized solution to transport equation : it is a solution such that

$$
\partial_{t} \beta(u(t, x))+b(t, x) \nabla \beta(u(t, x))=0 .
$$

for any suitable non-linearity $\beta$. Notice that (1.2) holds for smooth solutions, by an immediate application of the chain-rule. However, when the vector field is not smooth, we cannot expect any regularity of the solutions, so that (1.2) is a nontrivial request when made for all bounded distributional solutions. The renormalization property asserts that nonlinear compositions of the solution are again solutions, or alternatively that the chain-rule holds in this weak context. The overall result which motivates this definition is that, if the renormalization property holds, then solutions of (1.1) are unique and stable.

In the case $b$ has $W^{1,1}$ spatial regularity (together with a condition of boundedness on the divergence) the commutator lemma between smoothing convolution and weak solution can be proved and, as a consequence, all $L^{\infty}$ weak solutions are renormalized. Afters some intermediate results ( 4] and [5] ) the theory has been generalized by L. Ambrosio [1] to the case of only $B V$ regularity for b instead of $W^{1,1}$. See [2] and [7] for a nice review on that.

We consider the following stochastic transport equation

$$
\left\{\begin{array}{l}
\partial_{t} u(t, x)+\left(b(t, x)+\frac{d B_{t}}{d t}\right) \nabla u(t, x)=0, \\
\left.u\right|_{t=0}=u_{0},
\end{array}\right.
$$

$\left((t, x) \in U_{T}, \omega \in \Omega\right)$, where $U_{T}=[0, T] \times \mathbb{R}^{d}$, for $T>0$ be any fixed real number, $(d \in \mathbb{N}), b: \mathbb{R}_{+} \times \mathbb{R}^{d} \rightarrow \mathbb{R}^{d}$ is a given vector field, $B_{t}=\left(B_{t}^{1}, \ldots, B_{t}^{d}\right)$ is a standard Brownian motion in $\mathbb{R}^{d}$ and the stochastic integration is taken (unless otherwise mentioned) in the Stratonovich sense.

The Cauchy problem for the stochastic transport/continuity equation has taken great attention recently. F. Flandoli, M. Gubinelli and E. Priola in [11] obtained well-posedness of the stochastic problem for a bounded Hölder continuous drift term, with some integrability conditions on the divergence. E.Fedrizzi and F. Flandoli in [9] obtained a well-posedness result, in the class of local Sobolev solutions, under only some integrability conditions on 
the drift. There, it is only assumed that

$$
\begin{aligned}
b & \in L^{q}\left([0, T] ; L^{p}\left(\mathbb{R}^{d}\right)\right), \\
\text { for } \quad p, q \in[2, \infty), & \frac{d}{p}+\frac{2}{q}<1 .
\end{aligned}
$$

The well-posedness of the Cauchy problem (1.3) under condition (1.4) for measurable initial condition was also considered in [16] but with free divergence condition. In [3], using a technique based on the regularising effect observed on expected values of moments of the solution, well-posedness of (1.3) was obtained also for the limit cases of $p, q=\infty$ or when the inequality in (1.4) becomes an equality. In [10] the authors proved uniqueness when the field vectors $b \in L_{l o c}^{2}$ for a new class of solutions. Finally, we mention the paper [15] where the authors show uniqueness of the one-dimensional continuity equations when the drift is measurable and the linear growth.

In this paper we obtain a well-posedness result for unbounded vector fields. We prove existence, uniqueness and strong stability result for $W^{1, p}$ - solutions with unbounded locally Holder continuous drift and $\operatorname{div} b \in L_{l o c}^{q}$. The proofs are based on a careful analysis of the associated stochastic flow of characteristics and stochastic calculus techniques. The uniqueness result is an improvement of the condition considered in the seminar paper [11] where case bounded locally Holder drift was considered.

We would like to point that in the deterministic transport equation it is not possible to show existence in the class of $W^{1, p}$ - solutions. As showed

by Colombini, Luo and Rauch in [6], there exists an important example of $b \in L^{\infty} \cap W^{1, p},(\forall p<\infty)$, such that the propagation of the continuity in the deterministic transport equation is missing. That is to say, one may start with a continuous initial data, but the deterministic solution of the transport equation is not continuous. However, in the stochastic case we have the persistence property.

In fact, through of this paper, we fix a stochastic basis with a $d$-dimensional Brownian motion $\left(\Omega, \mathcal{F},\left\{\mathcal{F}_{t}: t \in[0, T]\right\}, \mathbb{P},\left(B_{t}\right)\right)$.

\section{$1.1 \quad$ Hypothesis}

Throughout this article we consider $1<p<\infty$. We assume 


\section{Hypothesis 1.1.}

$$
b \in C^{\theta}\left(\mathbb{R}^{d}, \mathbb{R}^{d}\right),
$$

and

$$
\operatorname{div} b(x) \in L_{l o c}^{q}\left(\mathbb{R}^{d}\right) \text { with } \frac{1}{p}+\frac{1}{q}=1 .
$$

Moreover, the initial condition is taken to be

$$
u_{0} \in W^{1,2 p}\left(\mathbb{R}^{d}\right) .
$$

\section{Results.}

\section{$2.1 \quad$ Notations}

For any $\theta \in(0,1)$, we denoted $C^{\theta}\left(\mathbb{R}^{d} ; \mathbb{R}^{d}\right), d \geq 1$ the space of the field vectors $f: \mathbb{R}^{d} \rightarrow \mathbb{R}^{d}$ such that

$$
[f]_{\theta}:=\sup _{x \neq y,|x-y| \leq 1} \frac{|f(x)-f(y)|}{|x-y|^{\theta}}<\infty .
$$

The space $C^{\theta}\left(\mathbb{R}^{d} ; \mathbb{R}^{d}\right)$ is the Banach space with the norm

$$
\|f\|_{\theta}=\left\|(1+|\cdot|)^{-1} f\right\|_{\infty}+[f]_{\theta} .
$$

Let us start by setting the notation used and then recalling the main results. For $0 \leq s \leq t$ and $x \in \mathbb{R}^{d}$, consider the following stochastic differential equation in $\mathbb{R}^{d}$

$$
X_{s, t}(x)=x+\int_{s}^{t} b\left(X_{s, r}(x)\right) d r+B_{t}-B_{s},
$$

where $X_{s, t}(x)=X(s, t, x)$, also $X_{t}(x)=X(0, t, x)$. Under condition (1.5), $X_{s, t}(x)$ is a stochastic flow of $C^{1}$-diffeomorphism( see [12]) Moreover, the inverse $Y_{s, t}(x):=X_{s, t}^{-1}(x)$ satisfies the following backward stochastic differential equation

$$
Y_{s, t}(x)=x-\int_{s}^{t} b\left(Y_{r, t}(x)\right) d r-\left(B_{t}-B_{s}\right),
$$


for $0 \leq s \leq t$, see [11]. We denote by $\phi_{s, t}$ the flow associeted to $X_{s, t}$ and $\psi_{s, t}$ its inverse.

We also recall the important results in [12] : Let $b_{n} \in C^{\theta}\left(\mathbb{R}^{d}, \mathbb{R}^{d}\right)$, and let $\phi_{s, t}^{n}$ be the corresponding stochastic flows, assume that $\left\|b_{n}-b\right\|_{C_{b}^{\theta}\left(\mathbb{R}^{d}, \mathbb{R}^{d}\right)} \rightarrow 0$ as $n \rightarrow \infty$, then for all $p \geq 1$ we have

$$
\begin{gathered}
\lim _{n \rightarrow \infty} \sup _{x \in \mathbb{R}^{d}} \sup _{s \in[0, T]} \mathbb{E}\left[\sup _{t \in[s, T]}\left|D \phi_{s, t}^{n}(x)-D \phi_{s, t}(x)\right|^{p}\right]=0 \\
\lim _{n \rightarrow \infty} \sup _{x \in \mathbb{R}^{d}} \sup _{s \in[0, T]} \mathbb{E}\left[\sup _{t \in[s, T]}\left|\frac{\phi_{s, t}^{n}(x)-\phi_{s, t}(x)}{1+|x|}\right|^{p}\right]=0
\end{gathered}
$$

and

$$
\sup _{n} \sup _{x \in \mathbb{R}^{d}} \sup _{s \in[0, T]} \mathbb{E}\left[\sup _{t \in[s, T]}\left|D \phi_{s, t}^{n}(x)\right|^{p}\right]<\infty .
$$

The same results are valid for the backward flows $\psi_{s, t}^{n}$ and $\psi_{s, t}$ since are solutions of the same SDE driven by the drifts $-b_{n}$ and $-b$.

\subsection{Definition.}

The next definition tells us in which sense a stochastic process is a $W^{1, p_{-}}$ solution of (1.3). We denoted $C_{0}^{\infty}\left(\mathbb{R}^{d}\right)$ the space of the test functions with compact support. We denoted $\mu=e^{-|x|^{2}}$ the gaussian measure in $\mathbb{R}^{d}$.

Definition 2.1. A stochastic process $u \in L^{2 p}\left(\Omega \times[0, T] \times \mathbb{R}^{d}\right) \cap L^{p}(\Omega \times$ $\left.[0, T], W^{1, p}\left(\mathbb{R}^{d}\right), \mu\right)$ is called a $W^{1, p}$ - weak solution of the Cauchy problem (1.3) when: for any $\varphi \in C_{0}^{\infty}\left(\mathbb{R}^{d}\right)$, the real valued process $\int u(t, x) \varphi(x) d x$ has a continuous modification which is an $\mathcal{F}_{t}$-semimartingale, and for all $t \in[0, T]$, we have $\mathbb{P}$-almost surely

$$
\begin{aligned}
\int_{\mathbb{R}^{d}} u(t, x) \varphi(x) d x= & \int_{\mathbb{R}^{d}} u_{0}(x) \varphi(x) d x-\int_{0}^{t} \int_{\mathbb{R}^{d}} b^{i}(x) \partial_{i} u(s, x) \varphi(x) d x d s \\
& +\int_{0}^{t} \int_{\mathbb{R}^{d}} u(s, x) \partial_{i} \varphi(x) d x \circ d B_{s}^{i} .
\end{aligned}
$$


Remark 2.2. Using the same idea as in Lemma 13 [11], on can write the problem (1.3) in Itô form as follows. A stochastic process $u \in L^{2 p}(\Omega \times$ $\left.[0, T] \times \mathbb{R}^{d}\right) \cap L^{p}\left(\Omega \times[0, T], W^{1, p}\left(\mathbb{R}^{d}\right), \mu\right)$ is a weak $W^{1, p}$ - solution of the SPDE (1.3) iff for every test function $\varphi \in C_{0}^{\infty}\left(\mathbb{R}^{d}\right)$, the process $\int u(t, x) \varphi(x) d x$ has a continuous modification which is a $\mathcal{F}_{t}$-semimartingale and satisfies the following Itô' formulation

$$
\begin{gathered}
\int u(t, x) \varphi(x) d x=\int u_{0}(x) \varphi(x) d x \\
-\int_{0}^{t} \int b^{i}(x) \partial_{i} u(s, x) \varphi(x) d x d s \\
+\int_{0}^{t} \int \partial_{i} \varphi(x) u(s, x) d x d B_{s}^{i}+\frac{1}{2} \int_{0}^{t} \int \Delta \varphi(x) u(s, x) d x d s .
\end{gathered}
$$

\section{$2.3 \quad$ Existence.}

Proposition 2.3. We assume hypothesis (1.1). Then $u(t, x)=u_{0}\left(X_{t}^{-1}\right)$ is a $W^{1, p}$-solution of the equation (1.3).

Proof. Step 1 : Regular initial data. We assume that $u_{0} \in C_{0}^{\infty}\left(\mathbb{R}^{d}\right)$. Let $\left\{\rho_{\varepsilon}\right\}_{\varepsilon}$ be a family of standard symmetric mollifiers. We define the family of regularised coefficients as $b^{\epsilon}(x)=\left(b * \rho_{\varepsilon}\right)(x)$.

For any fixed $\varepsilon>0$, the classical theory of Kunita, see [13] or [14], provides the existence of a unique solution $u^{\varepsilon}$ to the regularised equation

$$
\left\{\begin{array}{l}
d u^{\varepsilon}(t, x)+\nabla u^{\varepsilon}(t, x) \cdot\left(b^{\varepsilon}(x) d t+\circ d B_{t}\right)=0, \\
\left.u^{\varepsilon}\right|_{t=0}=u_{0}
\end{array}\right.
$$

in terms of the (regularised) initial condition and the inverse flow $\left(\phi_{t}^{\varepsilon}\right)^{-1}$ associated to the equation of characteristics of (2.14), which reads

$$
d X_{t}=b^{\varepsilon}\left(X_{t}\right) d t+d B_{t}, \quad X_{0}=x .
$$

We denoted $\left(\phi_{t}^{\varepsilon}\right)^{-1}$ by $\psi^{\epsilon}$. If $u^{\varepsilon}$ is a solution of (2.14), it is also a weak solution, which means that for any test function $\varphi \in C_{c}^{\infty}\left(\mathbb{R}^{d}\right)$, $u^{\varepsilon}$ satisfies 
the following equation (written in Itô form)

$$
\begin{array}{r}
\int_{\mathbb{R}^{d}} u^{\varepsilon}(t, x) \varphi(x) d x=\int_{\mathbb{R}^{d}} u_{0}(x) \varphi(x) d x-\int_{0}^{t} \int_{\mathbb{R}^{d}} \partial_{i} u^{\varepsilon}(s, x) b^{i, \varepsilon}(x) \varphi(x) d x d s \\
+\int_{0}^{t} \int_{\mathbb{R}^{d}} u^{\varepsilon}(s, x) \partial_{x_{i}} \varphi(x) d x d B_{s}^{i}+\frac{1}{2} \int_{0}^{t} \int_{\mathbb{R}^{d}} u^{\varepsilon}(s, x) \Delta \varphi(x) d x d s .
\end{array}
$$

We claim that

$$
u_{0}\left(\psi_{t}^{\epsilon}\right) \rightarrow u_{0}\left(\psi_{t}\right), \quad \mathbb{P} \otimes d t \otimes d x-\text { a.e } \quad \Omega \times[0, T] \times B_{R},
$$

as $\epsilon \rightarrow 0$. In fact, we have

$$
\begin{gathered}
\lim _{\epsilon \rightarrow 0} \int_{0}^{T} \int_{B_{R}} \int_{\Omega}\left|\psi_{t}^{\epsilon}(x)-\psi_{t}(x)\right|^{p} \mathbb{P}(d w) d x d t \\
\leq(1+R)^{p} \lim _{\epsilon \rightarrow 0} \int_{0}^{T} \int_{B_{R}} \int_{\Omega} \frac{\left|\psi_{t}^{\epsilon}(x)-\psi_{t}(x)\right|^{p}}{(1+|x|)^{p}} \mathbb{P}(d w) d x d t \\
\leq T\left|B_{R}\right|(1+R)^{p} \lim _{\epsilon \rightarrow 0} \sup _{t \in[0, T]} \sup _{x \in B_{R}} \mathbb{E}\left[\frac{\left|\psi_{t}^{\epsilon}(x)-\psi_{t}(x)\right|^{p}}{(1+|x|)^{p}}\right]=0 .
\end{gathered}
$$

Therefore, the sequence $\left\{\psi^{\epsilon}\right\}$ converge to $\psi$ in $L^{p}\left(\Omega \times[0, T] \times B_{R}\right)$ as $\epsilon \rightarrow 0$. Thus there exist a subsequence $\psi^{\epsilon}$, such that

$$
\psi^{\epsilon} \rightarrow \psi, \quad \mathbb{P} \otimes d t \otimes d x-\text { a.e. } \quad \Omega \times[0, T] \times B_{R}
$$

Now, we have

$$
\begin{gathered}
\int_{0}^{t} \int_{\mathbb{R}^{d}} \partial_{i} u^{\epsilon}(s, x) b^{i, \epsilon}(x) \varphi(x) d x d s= \\
-\int_{0}^{t} \int_{\mathbb{R}^{d}} u^{\epsilon}(s, x) b^{i, \epsilon}(x) \partial_{i} \varphi(x) d x d s-\int_{0}^{t} \int_{\mathbb{R}^{d}} u^{\epsilon}(s, x) d i v b^{\epsilon}(x) \varphi(x) d x d s .
\end{gathered}
$$

By dominated convergence we obtain

$$
\int_{0}^{t} \int_{\mathbb{R}^{d}} u^{\epsilon}(s, x) b^{i, \epsilon}(x) \partial_{i} \varphi(x) d x d s \rightarrow \int_{0}^{t} \int_{\mathbb{R}^{d}} u(s, x) b^{i}(x) \partial_{i} \varphi(x) d x d s,
$$




$$
\begin{aligned}
\int_{0}^{t} \int_{\mathbb{R}^{d}} u^{\epsilon}(s, x) \operatorname{divb}^{\epsilon}(x) \varphi(x) d x d s & \rightarrow \int_{0}^{t} \int_{\mathbb{R}^{d}} u(s, x) \operatorname{divb}(x) \varphi(x) d x d s, \\
& \frac{1}{2} \int_{0}^{t} \int_{\mathbb{R}^{d}} u^{\epsilon}(s, x) \Delta \varphi(x) d x d s \rightarrow \frac{1}{2} \int_{0}^{t} \int_{\mathbb{R}^{d}} u(s, x) \Delta \varphi(x) d x d s .
\end{aligned}
$$

By stochastic dominated convergence theorem we obtain

$$
\int_{0}^{t} \int_{\mathbb{R}^{d}} u^{\epsilon}(s, x) \partial_{i} \varphi(x) d x d B_{s}^{i} \rightarrow \int_{0}^{t} \int_{\mathbb{R}^{d}} u(s, x) \partial_{i} \varphi(x) d x d B_{s}^{i} .
$$

Taking the limit in equation (2.15) we conclude that $u(t, x)=u_{0}\left(X_{t}^{-1}\right)$ verifies

$$
\begin{aligned}
\int_{\mathbb{R}^{d}} u(t, x) \varphi(x) d x & =\int_{\mathbb{R}^{d}} u_{0}(x) \varphi(x) d x \\
& -\int_{0}^{t} \int_{\mathbb{R}^{d}} \partial_{i} u(s, x) b^{i}(x) \varphi(x) d x d s \\
& +\int_{0}^{t} \int_{\mathbb{R}^{d}} u(s, x) \partial_{i} \varphi(x) d x d B_{s}^{i} \\
& +\frac{1}{2} \int_{0}^{t} \int_{\mathbb{R}^{d}} u(s, x) \Delta \varphi(x) d x d s .
\end{aligned}
$$

Moreover, we have

$$
\mathbb{E} \int\left|u_{0}\left(\psi_{t}\right)\right|^{2 p} d x=\int \mathbb{E}\left[J \phi_{t}\right]\left|u_{0}(x)\right|^{2 p} d x \leq C,
$$

and

$$
\begin{gathered}
\mathbb{E} \int\left|D u_{0}\left(\psi_{t}\right)\right|^{p} e^{-|x|^{2}} d x=\mathbb{E} \int\left|D u_{0}\left(\psi_{t}\right)\right|^{p}\left|D \psi_{t}\right|^{p} e^{-|x|^{2}} d x \\
\leq C\left(\mathbb{E} \int\left|D u_{0}\left(\psi_{t}\right)\right|^{2 p} e^{-|x|^{2}} d x+\mathbb{E} \int\left|D \psi_{t}\right|^{2 p} e^{-|x|^{2}} d x\right) \\
\leq \mathbb{E} \int\left|D u_{0}(x)\right|^{2 p}\left|J \phi_{t}\right| e^{-\left|\phi_{t}\right|^{2}} d x+C
\end{gathered}
$$




$$
\leq C \mathbb{E} \int\left|D u_{0}(x)\right|^{2 p} d x+C
$$

where we used (2.10) and (2.12). Thus we conclude $u(t, x)=u_{0}\left(\psi_{t}\right)$ that is a is a $W^{1, p}$-solution of the Cauchy problem (1.3).

Step 2: Irregular initial data. $u_{0} \in W^{2, p}\left(\mathbb{R}^{d}\right)$. Let $\left\{\rho_{\varepsilon}\right\}_{\varepsilon}$ be a family of standard symmetric mollifiers. Consider a nonnegative smooth cut-off function $\eta$ supported on the ball of radius 2 and such that $\eta=1$ on the ball of radius 1 . For every $\varepsilon>0$ introduce the rescaled functions $\eta_{\varepsilon}(\cdot)=$ $\eta(\varepsilon \cdot)$. We define the family of regular approximations of the initial condition $u_{0}^{\varepsilon}(x)=\eta_{\varepsilon}(x)\left(\left[u_{0}(\cdot) * \rho_{\varepsilon}(\cdot)\right](x)\right)$. By the step 1 we have that $u^{\varepsilon}(t, x)=u_{0}^{\varepsilon}\left(\psi_{t}\right)$ verifies

$$
\begin{aligned}
\int_{\mathbb{R}^{d}} u^{\epsilon}(t, x) \varphi(x) d x & =\int_{\mathbb{R}^{d}} u_{0}^{\varepsilon}(x) \varphi(x) d x \\
& -\int_{0}^{t} \int_{\mathbb{R}^{d}} \partial_{i} u^{\epsilon}(s, x) b(x) \varphi(x) d x d s \\
& +\int_{0}^{t} \int_{\mathbb{R}^{d}} u^{\epsilon}(s, x) \partial_{i} \varphi(x) d x d B_{s}^{i} \\
& +\frac{1}{2} \int_{0}^{t} \int_{\mathbb{R}^{d}} u^{\epsilon}(s, x) \Delta \varphi(x) d x d s
\end{aligned}
$$

We claim that

$$
u_{0}^{\varepsilon}(\psi) \rightarrow u_{0}(\psi) \text { in } L^{2 p}\left(\Omega \times[0, T] \times \mathbb{R}^{d}\right),
$$

In fact, doing the variable change $x=\psi_{t}$ we have

$$
\begin{aligned}
& \left.\int_{0}^{T} \int_{\mathbb{R}^{d}} E \mid u^{\epsilon}(s, x)-u(s, x)\right)\left.\right|^{2 p} d x d s \\
= & \int_{0}^{T} \int_{\mathbb{R}^{d}}\left|u_{0}^{\epsilon}(x)-u_{0}(x)\right|^{2 p} E\left|J \phi_{t}\right| d x d s \\
& \leq C \int_{0}^{T} \int_{\mathbb{R}^{d}}\left|u_{0}^{\epsilon}(x)-u_{0}(x)\right|^{2 p} d x d s
\end{aligned}
$$




$$
\leq C \int_{\mathbb{R}^{d}}\left|u_{0}^{\epsilon}(x)-u_{0}(x)\right|^{2 p} d x d s .
$$

where we used (2.10) and (2.12).

Now, we observe that

$$
\begin{gathered}
\mathbb{E} \int\left|D u_{0}\left(\psi_{t}\right)\right|^{p} e^{-|x|^{2}} d x=\mathbb{E} \int\left|D u_{0}\left(\psi_{t}\right)\right|^{p}\left|D \psi_{t}\right|^{p} e^{-|x|^{2}} d x \\
\leq C\left(\mathbb{E} \int\left|D u_{0}\left(\psi_{t}\right)\right|^{2 p} e^{-|x|^{2}} d x+\mathbb{E} \int\left|D \psi_{t}\right|^{2 p} e^{-|x|^{2}} d x\right) \\
\leq \mathbb{E} \int\left|D u_{0}(x)\right|^{2 p}\left|J \phi_{t}\right| e^{-\left|\phi_{t}\right|^{2}} d x+C \\
\leq C \mathbb{E} \int\left|D u_{0}(x)\right|^{2 p} d x+C
\end{gathered}
$$

where we used (2.10) and (2.12).

Then passing to the limit in (2.19) we conclude the that $u(t, x)=u_{0}\left(\psi_{t}\right)$ is a $W^{1, p}$-solution of equation (1.3).

\subsection{Uniqueness.}

In this section we prove the uniqueness result for $W^{1, p}$ - solutions.

Proposition 2.4. Assume hypothesis (1.1). If $u_{1}, u_{2} \in L^{p}\left([0, T] \times \Omega, W^{1, p}\left(\mathbb{R}^{d}\right)\right)$ are two weak $W^{1, p}$-solutions for the Cauchy problem (1.3), with the same initial data $u_{0} \in W^{1,2 p}\left(\mathbb{R}^{d}\right)$, then $u_{1}=u_{2}$ almost everywhere in $[0, T] \times \mathbb{R}^{d} \times \Omega$.

Proof. By linearity, it is enough to show that a weak $W^{1, p}$-solution $u$ with initial condition $u_{0}(x)=0$ vanishes identically. Let $\phi_{\varepsilon}, \phi_{\delta}$ be standard symmetric mollifiers. Thus $u_{\varepsilon}(t, \cdot)=u(t, \cdot) * \phi_{\varepsilon}$ verifies

$$
\begin{aligned}
\int_{\mathbb{R}^{d}} u_{\varepsilon}(t, z) d z & =-\int_{0}^{t} \int_{\mathbb{R}^{d}} \partial_{i} u(s, z) b^{i}(z) \phi_{\varepsilon}(y-z) d z d s \\
& +\int_{0}^{t} \int_{\mathbb{R}^{d}} u(s, z) \partial_{i} \phi_{\varepsilon}(y-z) d z \circ d B_{s}^{i} .
\end{aligned}
$$

One remarks that, for each $\varepsilon>0$ the equation for $u_{\varepsilon}$ is strong in the analytic sense. 
Now, we denote by $b^{\delta}$ the standard mollification of $b$, and let $X_{t}^{\delta}$ be the associated flow given by the SDE (2.8) replacing $b$ by $b^{\delta}$. Similarly, we consider $Y_{t}^{\delta}$, which satisfies the backward SDE (2.9).

Doing the variable $y=X_{t}^{\delta}$ we have

$$
\int_{\mathbb{R}^{d}} u_{\varepsilon}\left(t, X_{t}^{\delta}\right) \varphi(y) d y=\int_{\mathbb{R}^{d}} u_{\varepsilon}(t, y) J Y_{t}^{\delta} \varphi\left(Y_{t}^{\delta}\right) d y
$$

for each $t \in[0, T]$.

Now, we observe that by Itô formula or by Kunita [14] $v^{\delta}(t, x)=J Y_{t}^{\delta} \varphi\left(Y_{t}^{\delta}\right)$, satisfies the continuity equation in the classical sense, that is, it satisfies

$$
\left\{\begin{array}{l}
d v^{\delta}(t, x)+\operatorname{Div}\left(b^{\delta}(x) v^{\delta}(t, x)\right) d t+\nabla v^{\delta}(t, x) \circ d B_{t}=0 \\
\left.v^{\delta}\right|_{t=0}=\varphi(x)
\end{array}\right.
$$

Consider a nonnegative smooth cut-off function $\eta$ supported on the ball of radius 2 and such that $\eta=1$ on the ball of radius 1 . For each $R>0$ introduce the rescaled functions. $\eta_{R}(\cdot)=\eta\left(\frac{1}{R} \cdot\right)$

Then we may apply Itô's formula to the product of two semimartingales

$$
u_{\varepsilon}(t, x) v^{\delta}(t, x)
$$

and obtain that

$$
\begin{aligned}
\int_{\mathbb{R}^{d}} \eta_{R}(y) u_{\varepsilon}(t, y) & v^{\delta}(t, y) d y=-\int_{0}^{t} \int_{\mathbb{R}^{d}} \eta_{R}(y) u_{\varepsilon}(s, y) \operatorname{div}\left(b^{\delta}(y) v_{s}^{\delta}(s, y)\right) d y d s \\
& -\int_{0}^{t} \int_{\mathbb{R}^{d}} \eta_{R}(y) u_{\varepsilon}(s, y) \partial_{i}\left[v^{\delta}(s, y)\right] d y \circ d B_{s}^{i} \\
& -\int_{0}^{t} \int_{\mathbb{R}^{d}} \eta_{R}(y) v^{\delta}(s, y) \int_{\mathbb{R}^{d}} \partial_{i} u(s, z) b^{i}(z) \phi_{\varepsilon}(y-z) d z d y d s \\
& +\int_{0}^{t} \int_{\mathbb{R}^{d}} \eta_{R}(y) v^{\delta}(s, y) \int_{\mathbb{R}^{d}} u(s, z) \partial_{i} \phi_{\varepsilon}(y-z) d z d y \circ d B_{s}^{i} .
\end{aligned}
$$

We observe that 


$$
\begin{gathered}
\int_{0}^{t} \int_{\mathbb{R}^{d}} \eta_{R}(y) v^{\delta}(s, y) \int_{\mathbb{R}^{d}} u(s, z) \partial_{i} \phi_{\varepsilon}(y-z) d z d y \circ d B_{s}^{i} . \\
=\int_{0}^{t} \int_{\mathbb{R}^{d}} \eta_{R}(y) \partial_{i} v^{\delta}(s, y) u_{\varepsilon}(s, y) d y \circ d B_{s}^{i} \\
+\int_{0}^{t} \int_{\mathbb{R}^{d}} \partial_{i} \eta_{R}(y) v^{\delta}(s, y) u_{\varepsilon}(s, y) d y \circ d B_{s}^{i} .
\end{gathered}
$$

Now for $\delta>0$ fixed, passing to the limit as $\varepsilon$ goes to $0^{+}$, we obtain from the above equation

$$
\begin{aligned}
\int_{\mathbb{R}^{d}} u\left(t, X_{t}^{\delta}\right) & \eta_{R}\left(X_{t}\right) \varphi(x) d x \\
= & -\int_{0}^{t} \int_{\mathbb{R}^{d}} \eta_{R}(y) u(s, y) \operatorname{div}\left(b^{\delta}(y) v^{\delta}(s, y)\right) d y d s \\
& -\int_{0}^{t} \int_{\mathbb{R}^{d}} \eta_{R}(y) \partial_{i} u(s, y) b^{i}(y) v^{\delta}(s, y) d y d s \\
& +\int_{0}^{t} \int_{\mathbb{R}^{d}} \partial_{i} \eta_{R}(y) v^{\delta}(s, y) u_{\varepsilon}(s, y) d y \circ d B_{s}^{i} .
\end{aligned}
$$

Thus we deduce

$$
\begin{gathered}
\int_{\mathbb{R}^{d}} u\left(t, X_{t}^{\delta}\right) \eta_{R}\left(X_{t}\right) \varphi(x) d x \\
=\int_{0}^{t} \int_{\mathbb{R}^{d}} \eta_{R}(y) \partial_{i} u(s, y) b^{\delta}(y) v^{\delta}(s, y) d y d s \\
+\int_{0}^{t} \int_{\mathbb{R}^{d}} \partial_{i} \eta_{R}(y) u(s, y) b^{\delta}(y) v^{\delta}(s, y) d y d s \\
-\int_{0}^{t} \int_{\mathbb{R}^{d}} \eta_{R}(y) \partial_{i} u(s, y) b^{i}(y) v^{\delta}(s, y) d y d s \\
+\int_{0}^{t} \int_{\mathbb{R}^{d}} \partial_{i} \eta_{R}(y) v^{\delta}(s, y) u(s, y) d y \circ d B_{s}^{i} .
\end{gathered}
$$

Then, by (2.11) and applying the Dominated Convergence Theorem we pass to the limit as $\delta$ goes to $0^{+}$, to conclude that 


$$
\begin{gathered}
\int_{\mathbb{R}^{d}} u\left(t, X_{t}\right) \eta_{R}\left(X_{t}\right) \varphi(x) d x \\
=\int_{0}^{t} \int_{\mathbb{R}^{d}} \partial_{i} \eta_{R}(y) u(s, y) b(y) v(s, y) d y d s \\
+\int_{0}^{t} \int_{\mathbb{R}^{d}} \partial_{i} \eta_{R}(y) v(s, y) u(s, y) d y \circ d B_{s}^{i} .
\end{gathered}
$$

We observe that

$$
\begin{aligned}
& \left|\int_{0}^{t} \int_{\mathbb{R}^{d}} \partial_{i} \eta_{R}(y) u(s, y) b(y) v(s, y) d y d s\right| \\
& \leq C\left(\left.\left|\int_{0}^{t} \int_{R \leq|y| \leq 2 R}\right| u(s, y)\right|^{2 p} d y d s \mid\right)^{\frac{1}{2 p}}
\end{aligned}
$$

where used that $b$ is the linear growth, and

$$
\begin{gathered}
\left|\int_{\mathbb{R}^{d}} \partial_{i} \eta_{R}(y) v(s, y) u(s, y) d y\right| \\
\leq \frac{1}{R}\left(\left.\left|\int_{0}^{t} \int_{0}^{t} \int_{R \leq|y| \leq 2 R}\right| u(s, y)\right|^{2 p} d y d s \mid\right)^{\frac{1}{2 p}}
\end{gathered}
$$

Passing to the limit as $R \rightarrow \infty$ we obtain

$$
\int_{\mathbb{R}^{d}} u\left(t, X_{t}\right) \varphi(x) d x=0
$$

for each $\varphi \in C_{0}^{\infty}\left(\mathbb{R}^{d}\right)$, and $t \in[0, T]$.

Thus, we have

$$
\begin{aligned}
\int \mathbb{E}|u(t, x)|^{2 p} d x & =\int \mathbb{E}\left|u\left(t, X_{t}\left(Y_{t}\right)\right)\right|^{2 p} d x \\
& =\mathbb{E} \int J X_{t}\left|\eta_{R}\left(X_{t}\right) u\left(t, X_{t}\right)\right|^{2 p} d x=0
\end{aligned}
$$

where we have used (2.27) and that $X_{t}$ is a stochastic flows of diffeomorphism. Consequently, the thesis of our theorem is proved. 


\subsection{Strong Stability.}

To end up the well-posedness for the Cauchy problem (1.3), it remains to show the stability property for the solution with respect to the initial datum and respect to the drift term

Proposition 2.5. Assume cond. Let $\left\{u_{0}^{n}\right\}$ be any sequence, with $u_{0}^{n} \in$ $W^{1,2 p}\left(\mathbb{R}^{d}\right)(n \geq 1)$, strong converging in $W^{1,2 p}$ to $u_{0}$. Let $u(t, x), u^{n}(t, x)$ be the unique weak $W^{1, p}$-solution of the Cauchy problem (1.3), for respectively the initial data $u_{0}$ and $u_{0}^{n}$. Then $u^{n}(t, x)$ strong converge to $u(t, x)$ in $L^{2 p}\left(\Omega \times[0, T] \times \mathbb{R}^{d}\right)$ and in $L^{p}\left(\Omega \times[0, T], W^{1, p}\left(\mathbb{R}^{d}\right), \mu\right)$.

Proof. By existence and uniqueness theorems we have that

$$
u^{n}(t, x)=u_{0}^{n}\left(X_{t}^{-1}\right)
$$

and

$$
u(t, x)=u_{0}\left(X_{t}^{-1}\right) .
$$

Then we obtain

$$
\begin{aligned}
& \int_{0}^{T} \int_{\mathbb{R}^{d}} \mathbb{E}\left|u^{n}(t, x)-u(t, x)\right|^{2 p} d x \\
= & \int_{0}^{T} \int_{\mathbb{R}^{d}} \mathbb{E}\left|u_{0}^{n}\left(X_{t}^{-1}\right)-u_{0}\left(X_{t}^{-1}\right)\right|^{2 p} d x d s \\
= & \int_{0}^{T} \int_{\mathbb{R}^{d}} \mathbb{E}\left|u_{0}^{n}(x)-u_{0}(x)\right|^{2 p}\left|J X_{t}\right| d x d s \\
\leq & C \int_{\mathbb{R}^{d}}\left|u_{0}^{n}(x)-u_{0}(x)\right|^{2 p} d x
\end{aligned}
$$

where we used (2.10) and (2.12).

Now, we have

$$
\begin{gathered}
\int_{0}^{T} \int_{\mathbb{R}^{d}} \mathbb{E}\left|D\left[u^{n}(t, x)-u(t, x)\right]\right|^{p} e^{-|x|^{2}} d x d s \\
=\int_{0}^{T} \int_{\mathbb{R}^{d}} \mathbb{E}\left|D u_{0}^{n}\left(X_{t}^{-1}\right)-D u_{0}\left(X_{t}^{-1}\right)\right|^{p}\left|D X_{t}^{-1}\right|^{p} e^{-|x|^{2}} d x d s
\end{gathered}
$$




$$
\begin{gathered}
\leq\left(\int_{0}^{T} \int_{\mathbb{R}^{d}} \mathbb{E}\left|D u_{0}^{n}\left(X_{t}^{-1}\right)-D u_{0}\left(X_{t}^{-1}\right)\right|^{2 p} e^{-|x|^{2}} d x d s\right)^{\frac{1}{2}} \\
\quad \times\left(\int_{0}^{T} \int_{\mathbb{R}^{d}} \mathbb{E}\left|D X_{t}^{-1}\right|^{2 p} e^{-|x|^{2}} d x d s\right)^{\frac{1}{2}} \\
\leq C\left(\int_{0}^{T} \int_{\mathbb{R}^{d}} \mathbb{E}\left|D u_{0}^{n}(x)-D u_{0}(x)\right|^{2 p} J X_{t} e^{-\left|X_{t}\right|^{2}} d x d s\right)^{\frac{1}{2}} \\
\leq C\left(\int_{\mathbb{R}^{d}}\left|D u_{0}^{n}(x)-D u_{0}(x)\right|^{2 p} d x\right)^{\frac{1}{2}}
\end{gathered}
$$

where we used Holder inqueality, (2.10) and (2.12).

Finally, from estimation (2.28) and (2.29) we conclude our proposition.

Proposition 2.6. Assume hypothesis 1.1 for $b$ and $b_{n}$ and $u_{0} \in C^{1, \alpha}\left(\mathbb{R}^{d}\right)$. Moreover, we also assume $\left\|b_{n}-b\right\|_{C_{b}^{\theta}\left(\mathbb{R}^{d}, \mathbb{R}^{d}\right)} \rightarrow 0$ as $n \rightarrow \infty$. Let $u(t, x)$, $u^{n}(t, x)$ be the unique weak $W^{1, p}$-solution of the Cauchy problem (1.3), for respectively the drift $b$ and $b_{n}$. Then $u^{n}(t, x)$ strong converge to $u(t, x)$ in $L^{p}\left(\Omega \times[0, T], W^{1, p}\left(\mathbb{R}^{d}\right), \mu\right)$.

Proof. From existence and uniqueness theorems we have that

$$
u^{n}(t, x)=u_{0}\left(X_{t}^{-1, n}\right)
$$

and

$$
u(t, x)=u_{0}\left(X_{t}^{-1}\right) .
$$

Then we obtain

$$
\begin{aligned}
& \int_{0}^{T} \int \mathbb{E}\left|u^{n}(t, x)-u(t, x)\right|^{2 p} e^{-|x|^{2}} d x d s \\
= & \int_{0}^{T} \int \mathbb{E}\left|u_{0}\left(X_{t}^{-1}\right)-u_{0}\left(X_{t}^{-1, n}\right)\right|^{2 p} e^{-|x|^{2}} d x d s \\
\leq & \int_{0}^{T} \int \mathbb{E}\left|X_{t}^{-1}-X_{t}^{-1, n}\right|^{\alpha} e^{-|x|^{2}} d x d s .
\end{aligned}
$$


From (2.11) we conclude that $u^{n} \rightarrow u$ in $L^{p}\left(\Omega \times[0, T] \times \mathbb{R}^{d}, \mu\right)$. We get

$$
\begin{gathered}
\int_{0}^{T} \int \mathbb{E}\left|D\left[u^{n}(t, x)-u(t, x)\right]\right|^{p} e^{-|x|^{2}} d x d s \\
=\int_{0}^{T} \int \mathbb{E}\left|D u_{0}\left(X_{t}^{-1, n}\right) D X_{t}^{-1, n}-D u_{0}\left(X_{t}^{-1}\right) D X_{t}^{-1}\right|^{p} e^{-|x|^{2}} d x d s \\
\leq \int_{0}^{T} \int \mathbb{E}\left|D u_{0}\left(X_{t}^{-1, n}\right) D X_{t}^{-1, n}-D u_{0}\left(X_{t}^{-1}\right) D X_{t}^{-1, n}\right|^{p} e^{-|x|^{2}} d x d s \\
+\int_{0}^{T} \int_{\mathbb{R}^{K}} \mathbb{E}\left|D u_{0}\left(X_{t}^{-1}\right) D X_{t}^{-1, n}-D u_{0}\left(X_{t}^{-1}\right) D X_{t}^{-1}\right|^{p} e^{-|x|^{2}} d x d s
\end{gathered}
$$

We have

$$
\begin{gathered}
\int_{0}^{T} \int \mathbb{E}\left|D u_{0}\left(X_{s}^{-1, n}\right) D X_{t}^{-1, n}-D u_{0}\left(X_{s}^{-1}\right) D X_{t}^{-1, n}\right|^{p} e^{-|x|^{2}} d x d s \\
\leq\left(\int_{0}^{T} \int\left(\mathbb{E}\left|D u_{0}\left(X_{s}^{-1, n}\right)-D u_{0}\left(X_{s}^{-1}\right)\right|^{2 p}\right) e^{-|x|^{2}} d x d s\right)^{\frac{1}{2}} \\
\times\left(\int_{0}^{T} \int\left|D X_{s}^{-1, n}\right|^{2 p} e^{-|x|^{2}} d x d s\right)^{\frac{1}{2}} \\
\leq C\left(\int_{0}^{T} \int\left(\mathbb{E}\left|X_{s}^{-1, n}-X_{s}^{-1}\right|^{2 p \alpha} e^{-|x|^{2}} d x d s\right)^{\frac{1}{2}}\right.
\end{gathered}
$$

where we used (2.10) and (2.12) . From (2.11) we conclude that

$$
\int_{0}^{T} \int \mathbb{E}\left|D u_{0}\left(X_{t}^{-1, n}\right) D X_{t}^{-1, n}-D u_{0}\left(X_{t}^{-1}\right) D X_{t}^{-1, n}\right|^{p} e^{-|x|^{2}} d x \rightarrow 0
$$

as $n \rightarrow \infty$.

Now, we obtain

$$
\int_{0}^{T} \int \mathbb{E}\left|D u_{0}\left(X_{t}^{-1}\right) D X_{t}^{-1, n}-D u_{0}\left(X_{t}^{-1}\right) D X_{t}^{-1}\right|^{p} e^{-|x|^{2}} d x d s
$$




$$
\begin{gathered}
\leq\left(\int_{0}^{T} \int \mathbb{E}\left|D u_{0}\left(X_{t}^{-1}\right)\right|^{2 p} e^{-|x|^{2}} d x d s\right)^{\frac{1}{2}} \\
\times\left(\int_{0}^{T} \int\left(\mathbb{E}\left|D X_{t}^{-1, n}-D X_{t}^{-1}\right|^{2 p} e^{-|x|^{2}} d x d s\right)^{\frac{1}{2}}\right. \\
\leq\left(\int_{0}^{T} \int\left(\mathbb{E}\left|D X_{t}^{-1, n}-D X_{t}^{-1}\right|^{2 p} e^{-|x|^{2}} d x d s\right)^{\frac{1}{2}}\right.
\end{gathered}
$$

where we used (2.10) and (2.12). From (2.10) we deduce that

$$
\int_{0}^{T} e^{-|x|^{2}} \int \mathbb{E}\left|D u_{0}\left(X_{t}^{-1}\right) D X_{t}^{-1, n}-D u_{0}\left(X_{t}^{-1}\right) D X_{t}^{-1}\right|^{p} d d x d s d x \rightarrow 0
$$

as $n \rightarrow \infty$.

Finally from (2.30), (2.31) and (2.32) we conclude our proposition.

\section{Acknowledgements}

Christian Olivera is partially supported by CNPq through the grant 460713/20140 and FAPESP by the grants 2015/04723-2 and 2015/07278-0.

\section{References}

[1] L. Ambrosio, Transport equation and Cauchy problem for BV vector fields, Invent. Math., 158, 227-260, 2004.

[2] L. Ambrosio G. Crippa Continuity equations and ODE fows with nonsmooth velocity, Lecture Notes of a course given at HeriottWatt University, Edinburgh. Proceeding of the Royal Society of Edinburgh, Section A: Mathematics. In press.

[3] L. Beck, F. Flandoli, M. Gubinelli and M. Maurelli, Stochastic ODEs and stochastic linear PDEs with critical drift: regularity, duality and uniqueness . Preprint available on Arxiv: 1401-1530, 2014. 
[4] F. Colombini, N. Lerner Sur les champs de vecteurs peu reguliers. Seminaire Equations aux Derivees Partielles, Exp. No. XIV, Ecole Polytech., Palaiseau, 2001.

[5] F. Colombini, N. Lerner, Uniqueness of continuous solutions for $B V$ vector fields, Duke Math. J., 111, 357-384, 2002.

[6] F. Colombini, T. Luo, J. Rauch, Nearly Lipschitzean divergence-free transport propagates neither continuity nor BV regularity, Commun. Math. Sci., 2, 207-212, 2004.

[7] C. De Lellis, Ordinary differential equations with rough coefficients and the renormalization theorem of Ambrosio, Bourbaki Seminar, Preprint, 1-26, 2007.

[8] R. DiPerna and P.L. Lions, Ordinary differential equations, transport theory and Sobolev spaces. Invent. Math., 98, 511-547, 1989.

[9] E. Fedrizzi and F. Flandoli, Noise prevents singularities in linear transport equations. Journal of Functional Analysis, 264, 1329-1354, 2013.

[10] E. Fedrizzi, W. Neves, C. Olivera. (2014) On a class of stochastic transport equations for $L_{l o c}^{2}$ vector fields, to appears in the Annali della Scuola Normale Superiore di Pisa, Classe di Scienze., arXiv:1410.6631v2.

[11] F. Flandoli, M. Gubinelli and E. Priola, Well-posedness of the transport equation by stochastic perturbation. Invent. Math., 180, 1-53, 2010.

[12] F. Flandoli, M. Gubinelli, E. Priola, Flow of diffeomorphisms for SDEs with unbounded Hölder continuous drift, Bulletin des Sciences Mathematiques, 134, 405-422, 2010.

[13] H. Kunita, Stochastic flows and stochastic differential equations. Cambridge University Press, 1990.

[14] H. Kunita, First order stochastic partial differential equations. In: Stochastic Analysis, Katata Kyoto, North-Holland Math. Library, 32, 249-269, 1984. 
[15] David A.C. Mollinedo and C. Olivera.(2017) Stochastic continuity equation with non-smooth velocity, to appears Annali di Matematica Pura ed Applicata , Doi : 10.1007/s10231-017-0633-8 .

[16] W. Neves and C. Olivera, Wellposedness for stochastic continuity equations with Ladyzhenskaya-Prodi-Serrin condition. NoDEA. Nonlinear Differential Equations and Applications, 22, 1-16, 2015. 